\title{
Temporal overlap of carnivorous mammal community and their prey in Khao Ang Rue Nai Wildlife Sanctuary, Chachoengsao Province, Thailand
}

\author{
RONGLARP SUKMASUANG ${ }^{\bullet}$, KHWANRUTAI CHARASPET, JITTIMA REONTIK, MANANYA PLA-ARD \\ Department of Forest Biology, Faculty of Forestry, Kasetsart University, Chatuchak District, Bangkok 10900, Thailand. \\ Tel.+66-579-0176, Fax. +66-942-8107. `email: mronglarp@gmail.com
}

Manuscript received: 30 December 2019. Revision accepted: 6 February 2020.

\begin{abstract}
Sukmasuang R, Charaspet K, Reontik J, Pla-ard M. 2020. Temporal overlap of carnivorous mammal community and their prey in Khao Ang Rue Nai Wildlife sanctuary, Chachoengsao Province, Thailand. Biodiversitas 21: 922-932. This study on the temporal overlap of the carnivorous community and their prey in Khao Ang Rue Nai Wildlife Sanctuary was conducted from March 2017 to February 2018. Camera traps were deployed systematically with a total of 4,463 trap nights. Fourteen carnivorous mammals were recorded, which were mainly present at night, with the exception of the dhole, small Indian mongoose, crab-eating mongoose, and yellow-throated marten. The clouded leopard's presence overlapped between day and night. Using the average $\operatorname{coefficient}$ overlap $(\Delta)$ between a carnivore, and the other carnivorous species, the leopard cat was found to have the highest $\Delta$ value, followed by the hog badger, Asiatic jackal, small Indian civet, Asian palm civet, large Indian civet, large spotted civet, Asiatic black bear, dhole, Malayan sun bear, yellow-throated marten, small Indian mongoose, crab-eating mongoose, and clouded leopard. The potential prey species that had the highest $\Delta$ with the carnivorous species, was the Siamese hare. This study shows the importance of preserving the carnivorous community within the area. An important threat is a likelihood that carnivorous species in the area may be exposed to external diseases from infected domestic animals when coming out to hunt in the communities surrounding the protected area.
\end{abstract}

Keywords: Camera trap, eastern forest complex, predator and prey, overlap package

\section{INTRODUCTION}

The rainforests are home to a complex diversity of animals, which include many carnivorous species located at the top of the trophic networks (Ridout and Linkie 2009). Carnivores are a particularly difficult species to study due to their ability to evade (Van Schaik and Grifthths 1996), with very few studies conducted on the interaction between carnivores and their prey in tropical areas. These studies are important to conduct, as they provide ecological data to help understand the components and mechanisms of the interactions within a dynamic and changing ecosystem (Hongliang et al. 2016). Previous studies have examined the activity and temporal overlap of wild species using the time recorded on photographs taken by camera traps (Laidlaw and Shaharuddin 1998; Kawanishi and Sunquist 2004; Di Bitetti et al. 2009; Di Bitetti et al. 2010; Monterroso et al. 2013; Sunarto et al. 2015) and via determining the temporal overlap of carnivorous species in tropical forests (Karanth and Sunquist 1995; Ray and Sunquist 2001; Scognamillo et al. 2003; Lynam et al. 2013). Nevertheless, studies are still lacking in the eastern forests of Thailand, including Khao Ang Rue Nai Wildlife Sanctuary (KARN), where the interaction of the temporal overlap of carnivores, and the time presence relationship with their prey species, have yet to be established. The information obtained, is essential to carry out conservation plans for both the animal species studied and the natural environments (Frey et al. 2017). Accordingly, the aims of this study were to (i) study the overall time presence of carnivorous mammals in the KARN area, (ii) study the activity cycle of each carnivorous species, and (iii) study the temporal overlap of carnivorous species, including their relationship with their prey by camera trapping in the area.

\section{MATERIALS AND METHODS}

\section{Study area}

Khao Ang Rue Nai (KARN) Wildlife Sanctuary, Chachoengsao Province, Thailand is located between longitude $101^{\circ} 35^{\prime}$ and $102^{\circ} 05^{\prime} \mathrm{E}$, and between latitude $13^{\circ}$ and $13^{\circ} 30^{\prime} \mathrm{N}$, with a total area of approximately 1,030 $\mathrm{km}^{2}$. It is a large dry evergreen forest, which was once connected to Cambodia, and is one of the world's important protected areas for both wild species and their habitats. It is now connected to 7 protected areas, adding to a total of $1,705 \mathrm{~km}^{2}$. KARN is the largest most important protected area in the east of Thailand. It is home to at least 531 species of vertebrates including 290 species of birds, 122 species of mammals, 90 species of reptiles, 29 species of amphibians and at least 47 species of fish. The terrain of the sanctuary is moderately sloping and was once encroached before the evacuation of humans out of the area due to Thai government recognizes the importance of preserving this forest area for conservation rather than changing it to agricultural land and human settlement. The southwestern, northeastern and southeastern parts have high mountains, and the elevation of the area varies from 30-802 meters above sea level. The highest peak is in the 
southeast, called Khao Sib Ha Chan, which reaches 802 meters above sea level. The plant communities are mainly dry evergreen forest with only little areas of mixed deciduous, dry dipterocarp and grassland (Department of National Parks Wildlife and Plant Conservation: DNP 2018). The study was conducted in the upper and middle parts of KARN, covering an area of $88.12 \mathrm{~km}^{2}$ when drawing a line connecting the outer camera trap locations, or when adding the buffer area with a radius of $1 \mathrm{~km}$ from the border (e.g. Kelly and Holub 2008) because of the movement of wildlife around the area.

\section{Procedures}

Camera traps were placed in 58 locations in Khao Ang Rue Nai Wildlife Sanctuary, in the Chachoengsao Province. The cameras were deployed from March 2017 to February 2018, adding up to a total of 4,463 trap nights; the details are as follows. (i) The 1: 50,000 topographic map was divided into $1 \mathrm{~km}^{2}$ grid. One camera trap was placed per one grid (Gupta et al. 2009; Jenks et al. 2011; Siripattaranukul et al. 2015a, b). Cameras were set up in 15-20 grids and left for 60 days in each location before moving to a new location (Figure 1). Each camera location was at least $500 \mathrm{~m}$ apart to enable independence of the photographs in each grid, and to reduce the possibility of recording the same animal across several cameras (Jenks et al. 2012). (ii) Camera trap location selection was considered from the suitability of the area, e.g., animal trails or signs of carnivorous species. Information around the location was collected including the plant community, roads, patrol routes, permanent water sources, salt licks, ponds and ranger stations (Prayoon et al. 2012; Lynam et al. 2013; Wongchoo et al. 2013; Siripattaranukul et al. 2015a,b). (iii) Camera traps were set up approximately 30$40 \mathrm{~cm}$ from the ground, 3-4 m away from the target point (Chutiponget al.2014a,b) or following the suitability of the area. The cameras were set to work 24 hours and take 3 consecutive photographs, 10 seconds apart, after the sensor was triggered (Network 2008). The cameras were placed for 60 days and then moved to new locations, which were recorded with a GPS tracker. (iv) The photographs taken by the camera traps were imported into a computer and classified in the Camera Trap Manager Program (Zaragozí et al. 2015) and added to Microsoft Excel for data analysis.

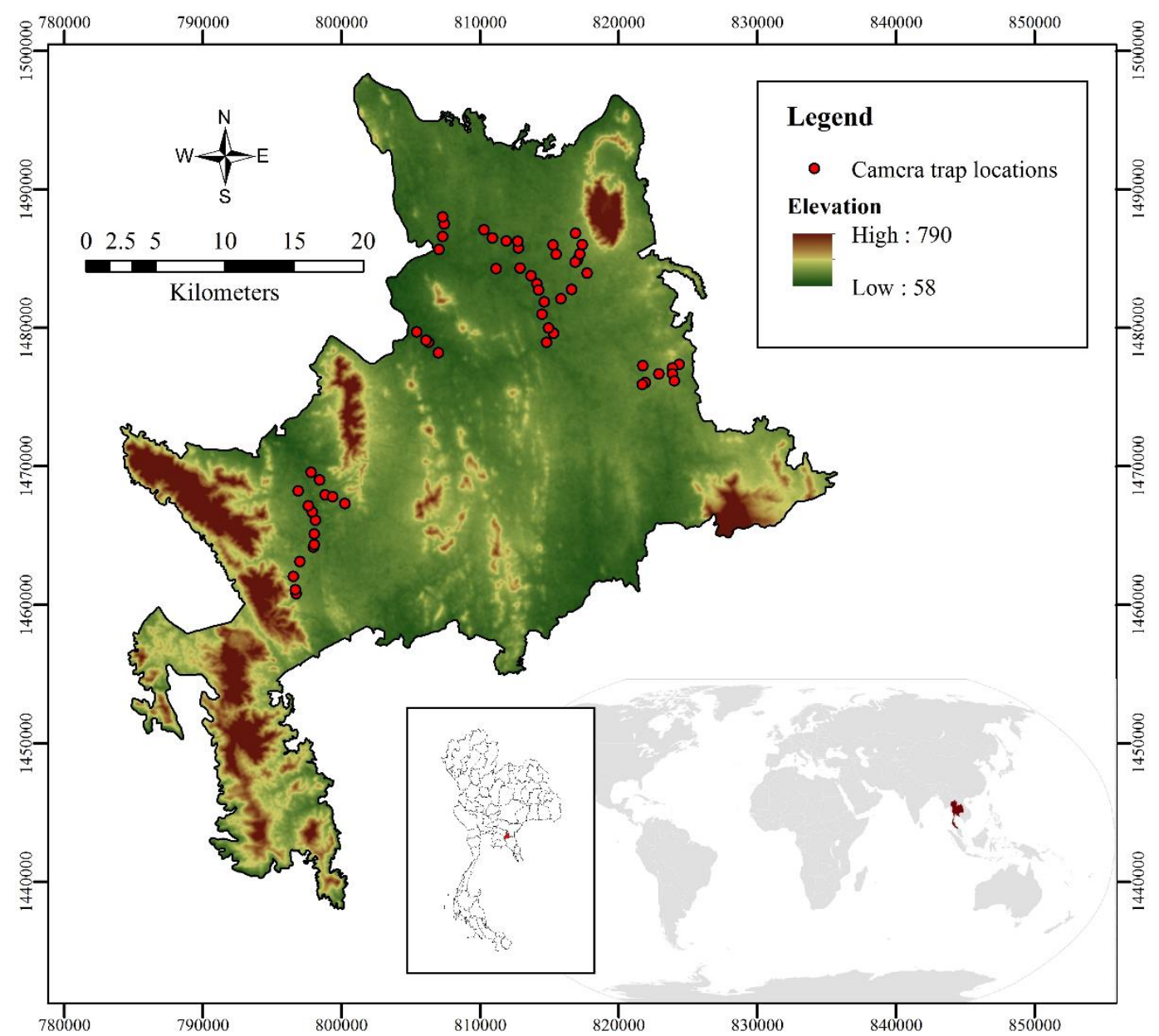

Figure 1. Map of Khao Ang Rue Nai Wildlife Sanctuary and the camera trap locations ( $n=58$ with 4,463 trap nights in total) 


\section{Data analysis}

(i) The carnivores were classified using their common and scientific names, following Lekagul and McNeely (1988). Only photographs that could be clearly identified with the date and time on the photograph were used. Photographs with more than one carnivore were counted as 1 incidence of each (Jenks et al. 2011), and were considered to be photos or incidences that were independent of each other. The criterion for independence of animal photographs used, included (1) consecutive photographs of different animals, which could be the same or different species, (2) consecutive photographs of the same animal of the same species that were taken more than 30 minutes apart, and (3) nonconsecutive photographs of the same animal of the same species (O'Brien et al. 2003). (ii) The time data from the photographs were summarised by compiling the data and classifying by species. The time period was divided into morning from 06:01-17:59 hrs and night between 18:00-06:00 hrs (Azlan et al. 2009). The data were then used to produce activity cycle graphs for carnivorous species and prey species, respectively. (iii) Time was divided into 24 hours. The activity periods of each species were classified from the photographs and divided into 2 periods, morning from 06:01-17:59 hrs and night between 18:00-06:00 hrs (Azlan and Sharma 2006; Azlan et al. 2009). The percentage of each time period for each species was calculated and the animals were divided into 5 groups following the method by Van Schaik and Griffiths (1996): if the number of photographs at night was more than $85 \%$, the species was classified as strongly nocturnal, if the number of photographs at night was between $61-84 \%$, the species was classified as mostly nocturnal, animals photographed at night and day time in between $40-60 \%$, were classified as cathemeral, species photographed during the day between $61-84 \%$, were classified as mostly diurnal, and animals with a number of day time photographs of more than $85 \%$, were classified as strongly diurnal. (iv) Calculating the style of the temporal overlap on the photographs between carnivorous species and prey species was performed using the method developed by Ridout and Linkie (2009), who used kernel density estimation (Fernandez-Duran 2004) to test the degree of the temporal overlap of the 2 species studied; called the coefficient of overlap $(\Delta)$. This is related to the area under the graph, or curve that shows the time of presence of both species together. For the function of the minimum density in each area of the 2 species compared, $\Delta$ $=1$ means that the activity overlaps, while $\Delta=0$ means no activity overlap was found. Calculating the confidence of $\Delta$ was performed using 500 bootstraps with $\mathrm{R}$ program version 2.11.1 ( $\mathrm{R}$ Development Core Team 2009) following Linkie and Ridout (2011).

\section{RESULTS AND DISCUSSION}

\section{Diel activity patterns}

From studying the activity cycle of the 14 species of carnivores, from 782 photographs, it was found that the carnivores that were in the strongly nocturnal group were
Asiatic black bears, Malayan sun bears, large Indian civets, large-spotted civets, and Asian palm civets. The group that was mostly nocturnal included Asiatic jackals, hog badgers, small Indian civets, and leopard cats. The cathemeral group included the clouded leopard. The mostly diurnal group included the dhole, and the strongly diurnal group included the yellow-throated marten, small Indian mongoose, and crab-eating mongoose, as shown in Table 1.

\section{Temporal overlap of carnivorous species}

In studying the activity cycles, from the overlapping areas of the two carnivorous species, the carnivore that had the highest temporal overlap with the Asiatic jackal was the small Indian civet, with a coefficient of overlap $(\Delta)$ value of 0.83 , followed by the leopard cat $(0.81)$, hog badger (0.76), large-spotted civet (0.74), Asian palm civet (0.73), large Indian civet (0.72), Asiatic black bear (0.63), dhole (0.51), Malayan sun bear (0.37), yellow-throated marten $(0.36)$, crab-eating mongoose $(0.33)$, small Indian mongoose (0.26) and clouded leopard (0.13).

The carnivore with the highest temporal overlap with the dhole was the yellow-throated marten, with a $\Delta$ value of 0.74 , followed by the crab-eating mongoose $(0.70)$, small Indian mongoose (0.62), Asiatic jackal (0.51), leopard cat (0.44), small Indian civet (0.41), yellow-throated marten (0.35), hog badger (0.33), large-spotted civet (0.27), large Indian civet (0.26), clouded leopard (0.23) and Asiatic black bear (0.14).

The carnivore with the highest temporal overlap with the Asiatic black bear was the large Indian civet, with a $\Delta$ value of 0.82 , followed by hog badger $(0.78)$, large-spotted civet (0.74), small Indian civet (0.70), leopard cat (0.62), and yellow-throated marten (0.46).

The carnivore with the highest temporal overlap with the Malayan sun bear, was the large-spotted civet and hog badger, with $\Delta$ values of 0.51 for both species, followed by the small Indian civet (0.50), leopard cat (0.48), large Indian civet (0.46), large-spotted civet (0.39), and clouded leopard (0.34). The carnivore with the highest temporal overlap with the clouded leopard was the Malayan sun bear, with a $\Delta$ value of 0.34 , followed by dhole, largespotted civet, small Indian mongoose, and leopard cat, all having the same $\Delta$ value of 0.23 . The details are shown in Table 2.

\section{Temporal overlap between carnivorous mammals and prey species}

In studying the coefficient of overlap between the time recorded from photographs taken between carnivores and their prey, it was found that when comparing important species, the species which had the highest activity overlap with the Asiatic jackals, was the Siamese hare, with a $\Delta$ value of 0.72 . This was followed by the murid rodent (0.70), Sambar deer (0.65), common muntjac (0.64), gaur (0.63), banteng (0.63), lesser mouse-deer (0.62), wild boar $(0.58)$ and pig-tailed macaque (0.36), respectively. The species with the highest activity overlap with dholes was the wild boar, with a $\Delta$ value of 0.88 , followed by the common muntjac (0.80), lesser mouse-deer (0.80), pigtailed macaque (0.75), gaur (0.42), banteng (0.41), Sambar 
deer (0.39), Siamese hare (0.40) and murid rodent (0.24). The species with the highest activity overlap with Asiatic black bear was the murid rodent, with a $\Delta$ value of 0.87 , followed by Sambar deer (0.73), Siamese hare (0.66), gaur and banteng (equal values of 0.47 ), common muntjac (0.29), lesser mouse-deer (0.28), wild boar (0.22) and pigtailed macaque (0.04). The species with the highest activity overlap with the Malayan sun bear was the Sambar deer, with a $\Delta$ value of 0.64 , followed by Siamese hare $(0.56)$, gaur (0.49), lesser mouse-deer (0.48), murid rodent (0.46), banteng (0.46), wild boar (0.36), common muntjac $(0.37)$ and pig-tailed macaque (0.17). The species with the highest activity overlap with clouded leopard was the gaur, with a $\Delta$ value of 0.47 , followed by banteng (0.45), Sambar deer (0.32), Siamese hare $(0.31)$, lesser mouse-deer $(0.30)$, pigtailed macaque $(0.17)$, common muntjac $(0.17)$, wild boar (0.16) and murid rodent (0.11); (Table 3$)$.

The relationship between the presence of carnivorous species and prey species showed that the average $\Delta$ of all species from the camera traps was 0.50 (see Table 3 ). The carnivore that had the highest $\Delta$ when compared to all species of prey was the Asiatic jackal and leopard cat, which both had $\Delta$ values of 0.61 , followed by the small Indian civet (0.59), Asian palm civet (0.58), dhole (0.57), and large Indian civet (0.56). An example of the $\Delta s$ in carnivorous and prey species are shown in Figure 2.

In examining the relationship between the time of presence of temporal overlap of each prey species with carnivores, it was found that that the highest $\Delta$ value of the prey species was the Siamese hare $(0.58)$, followed by the Sambar deer (0.56), murid rodent (0.53), gaur (0.52), banteng (0.51), lesser mouse-deer (0.50), common muntjac (0.48), wild boar (0.46) and pig-tailed macaque (0.36).

\section{Discussion}

The results of this study confirm the presence of 14 species of carnivorous mammals in the study area, which were mostly present at night (9 out of the 14 species). The strongly nocturnal species included the Asiatic black bear, Malayan sun bear, small Indian civet, large Indian civet, large-spotted civet, and Asian palm civet. The majority of nocturnal species included the Asiatic jackal, hog badger, and leopard cat. Four species were found to be diurnal, the yellow-throated marten, small Indian mongoose, and crabeating mongoose were all strongly diurnal, the dhole was mostly diurnal, and only one species, the clouded leopard, was cathemeral (see Table 1). The number of carnivorous species found in the area was lower than previously reported in Chhep Wildlife Sanctuary, which found 16 species, including leopards and jungle cats (Suzuki et al. 2017). Similarly, we observed a lower number of species compared with a study undertaken in the Cardamom Mountains, which included wetlands and hill forest areas (Holden and Neang 2009). The authors identified 15 carnivorous species, with the additional species in their study being the hairy-nosed otter, smooth-coated otter, and oriental small-clawed otter, respectively. Nevertheless, a greater number of carnivorous species were identified compared to a study conducted in Phnom Prich Wildlife Sanctuary, which only found 13 species (Gray and Phan 2011).

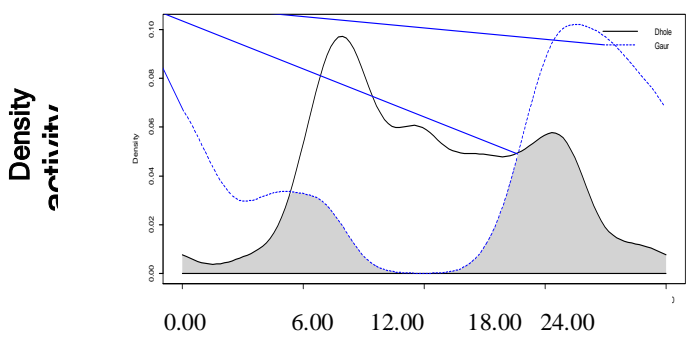

1. Dhole $(\mathrm{n}=99)$ and gaur $(\mathrm{n}=155) \Delta=42.13$

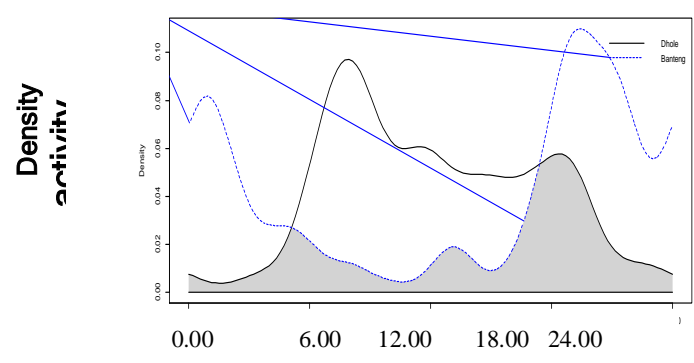

2. Dhole ( $\mathrm{n}=99)$ and banteng $(\mathrm{n}=117) \Delta=40.75$

Figure 2. Example of diel activity patterns from camera-trap data. Kernel density functions were used to depict dhole and some prey species activity, which was sampled via camera trapping during March 2017 and February 2018, in Khao Ang Rue Nai Wildlife Sanctuary. The overlap coefficient $(\Delta)$ is the area under the minimum of the two density estimates (denoted in grey)

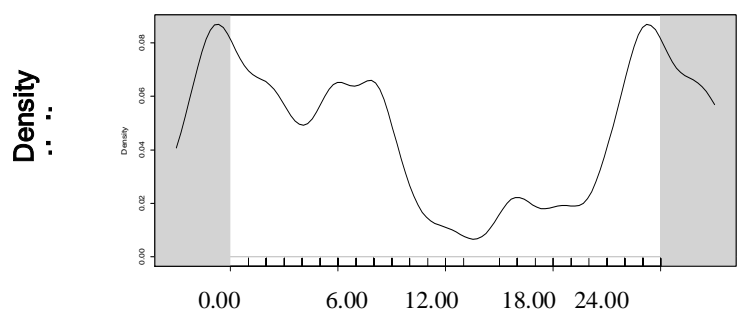

1. Asiatic jackal $(n=136)$

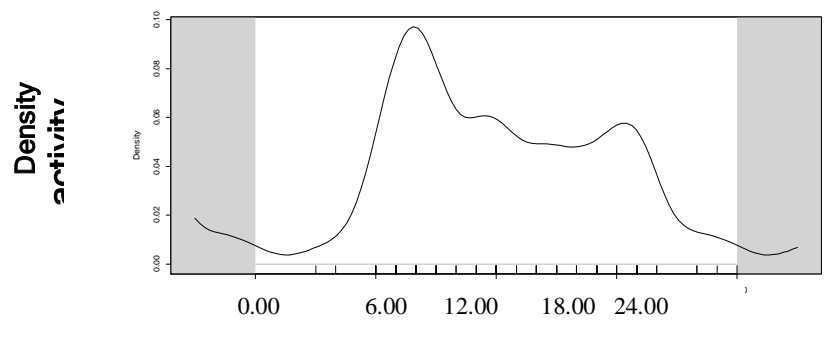

2. Dhole $(n=99)$

Figure 3. Kernel-density estimates of daily activity patterns of example carnivorous mammal species using camera trap data during March 2017 and February 2018, in Khao Ang Rue Nai Wildlife Sanctuary, Chachoengsao Province, Thailand 
Table 1. Number of detections in day time and night time for categorizing the cathemeral, nocturnal and diurnal periods, shown by their availability, of the carnivores in Khao Ang Rue Nai Wildlife Sanctuary, Chachoengsao Province, Thailand

\begin{tabular}{|c|c|c|c|c|c|c|c|}
\hline Family, scientific name & Common name & $\begin{array}{c}\text { Pictures in } \\
\text { day time }\end{array}$ & $\%$ & $\begin{array}{l}\text { Pictures in } \\
\text { night time }\end{array}$ & $\%$ & $\mathbf{N}^{1}$ & Periods $^{2}$ \\
\hline \multicolumn{8}{|l|}{ Canidae } \\
\hline Canis aureus & Asiatic Jackal & 28 & 20.59 & 108 & 79.41 & 136 & $\mathrm{Mn}$ \\
\hline Cuon alpinus & Dhole & 76 & 76.77 & 23 & 23.23 & 99 & Md \\
\hline \multicolumn{8}{|l|}{ Ursidae } \\
\hline Ursus thibetanus & Asiatic black bear & 0 & 0 & 2 & 100.00 & 2 & $\mathrm{Sn}$ \\
\hline Helarctos malayanus & Malayan sun bear & 0 & 0 & 3 & 100.00 & 3 & $\mathrm{Sn}$ \\
\hline \multicolumn{8}{|l|}{ Mustelidae } \\
\hline Martes flavigula & Yellow-throated marten & 12 & 100.00 & 0 & 0 & 12 & $\mathrm{Sd}$ \\
\hline Arctonyx collaris & Hog badger & 2 & 15.38 & 11 & 84.62 & 13 & $\mathrm{Mn}$ \\
\hline \multicolumn{8}{|l|}{ Herpestidae } \\
\hline Herpestes javanicus & Small Indian mongoose & 5 & 100.00 & 0 & 0 & 5 & $\mathrm{Sd}$ \\
\hline Herpestes urva & Crab-eating mongoose & 13 & 100.00 & 0 & 0 & 13 & $\mathrm{Sd}$ \\
\hline \multicolumn{8}{|l|}{ Viverridae } \\
\hline Viverricula indica & Small Indian Civet & 7 & 17.07 & 34 & 82.93 & 41 & $\mathrm{Mn}$ \\
\hline Viverra zibetha & Large Indian Civet & 0 & 0 & 14 & 100.00 & 14 & $\mathrm{Sn}$ \\
\hline Viverra megaspila & Large-spotted civet & 7 & 4.38 & 153 & 95.63 & 160 & $\mathrm{Sn}$ \\
\hline Paradoxurus hermaphroditus & Asian palm civet & 10 & 5.99 & 157 & 94.01 & 167 & $\mathrm{Sn}$ \\
\hline \multicolumn{8}{|l|}{ Felidae } \\
\hline Prionailurus bengalensis & Leopard cat & 21 & 18.26 & 94 & 81.74 & 115 & $\mathrm{Mn}$ \\
\hline Neofelis nebulosa & Clouded leopard & 1 & 50.00 & 1 & 50.00 & 2 & $\mathrm{C}$ \\
\hline Summary & & 182 & & 600 & & 782 & \\
\hline
\end{tabular}

Note: $1 \mathrm{~N}=$ Number of photos $2, \quad \mathrm{Sn}=$ strongly nocturnal,

$\mathrm{Mn}=$ mostly nocturnal, $\mathrm{C}=$ Cathemeral

$\mathrm{Md}=$ mostly diurnal,$\quad \mathrm{Sd}=$ strongly diurnal 
Table 2. The overlap coefficient $(\Delta)$ calculated using Kernel density functions of large carnivorous mammal species activity sampled via camera trapping during March 2017 to February 2018 , in Khao Ang Rue Nai Wildlife Sanctuary, (1=identical activity), with approximate $95 \%$ bootstrap confidence intervals in parentheses

\begin{tabular}{|c|c|c|c|c|c|c|c|c|c|c|c|c|c|c|}
\hline Species ${ }^{1}$ & DH & ABB & MSB & YTM & HB & SIC & LIC & LSC & APC & SIM & CEM & LC & CL & Average \\
\hline $\mathrm{AJ}$ & 0.51 & 0.63 & 0.37 & 0.36 & 0.76 & 0.83 & 0.72 & 0.74 & 0.73 & 0.26 & 0.33 & 0.81 & 0.13 & 0.55 \\
\hline & $(0.51-0.44)$ & $0.65-0.46)$ & $0.42-0.36)$ & $0.36-0.29)$ & $(0.82-0.75)$ & $(0.83-0.86)$ & $(0.83-0.71)$ & $(0.75-0.74)$ & $(0.73-0.73)$ & $(0.29-0.26)$ & $(0.33-0.27)$ & $(0.82-0.79)$ & $(0.21-0.12)$ & \\
\hline DH & & 0.14 & 0.35 & 0.74 & 0.33 & 0.41 & 0.26 & 0.27 & 0.31 & 0.62 & 0.70 & 0.44 & 0.23 & 0.40 \\
\hline & & $(0.19-0.13)$ & $(0.37-0.37)$ & $(0.74-0.73)$ & $(0.34-0.28)$ & $(0.41-0.32)$ & $(0.28-.008)$ & $(0.27-0.22)$ & $(0.31-0.29)$ & $(0.63-0.60)$ & $(0.70-0.69)$ & $(0.44-0.41)$ & $(0.29-0.23)$ & \\
\hline $\mathrm{ABB}$ & & & $\begin{array}{c}0.46 \\
(0.50-0.45)\end{array}$ & $\begin{array}{c}0.02 \\
(0.07-0.00\end{array}$ & $\begin{array}{c}0.78 \\
(0.80-0.76)\end{array}$ & $\begin{array}{c}0.70 \\
(0.70-0.61)\end{array}$ & $\begin{array}{c}0.82 \\
(1.07-0.82)\end{array}$ & $\begin{array}{c}0.74 \\
(0.75-0.74)\end{array}$ & $\begin{array}{c}0.75 \\
(1.24-0.74)\end{array}$ & $\begin{array}{c}0.01 \\
(0.05-0.00)\end{array}$ & $\begin{array}{c}0.02 \\
(0.07-0.00)\end{array}$ & $\begin{array}{c}0.62 \\
(0.63-0.55)\end{array}$ & $\begin{array}{c}0.02 \\
(0.05-0.00)\end{array}$ & 0.46 \\
\hline MSB & & & & $\begin{array}{c}0.13 \\
(0.18-0.08)\end{array}$ & $\begin{array}{c}0.51 \\
(0.53-0.51)\end{array}$ & $\begin{array}{c}0.50 \\
(0.53-0.48)\end{array}$ & $\begin{array}{c}0.46 \\
(0.49-0.42)\end{array}$ & $\begin{array}{c}0.39 \\
(0.43-0.28)\end{array}$ & $\begin{array}{c}0.51 \\
(0.52-0.37)\end{array}$ & $\begin{array}{c}0.20 \\
(0.23-0.20)\end{array}$ & $\begin{array}{c}0.14 \\
(0.19-0.14)\end{array}$ & $\begin{array}{c}0.48 \\
(0.51-0.44)\end{array}$ & $\begin{array}{c}0.34 \\
(0.34-0.33)\end{array}$ & 0.36 \\
\hline YTM & & & & & $\begin{array}{c}0.17 \\
(0.20-0.07)\end{array}$ & $\begin{array}{c}0.22 \\
(0.24-0.12)\end{array}$ & $\begin{array}{c}0.07 \\
(0.12-0.00)\end{array}$ & $\begin{array}{c}0.09 \\
(0.12-0.04)\end{array}$ & $\begin{array}{c}0.11 \\
(0.14-0.05)\end{array}$ & $\begin{array}{c}0.70 \\
(0.70-0.58)\end{array}$ & $\begin{array}{c}0.91 \\
(0.93-0.90)\end{array}$ & $\begin{array}{c}0.24 \\
(0.26-0.18)\end{array}$ & $\begin{array}{c}0.11 \\
(0.15-0.11)\end{array}$ & 0.31 \\
\hline $\mathrm{HB}$ & & & & & & $\begin{array}{c}0.83 \\
(0.84-0.83)\end{array}$ & $\begin{array}{c}0.81 \\
(0.84-0.80)\end{array}$ & $\begin{array}{c}0.81 \\
(0.82-0.78)\end{array}$ & $\begin{array}{c}0.85 \\
(0.95-0.83)\end{array}$ & $\begin{array}{c}0.17 \\
(0.19-0.07)\end{array}$ & $\begin{array}{c}0.16 \\
(0.20-0.07)\end{array}$ & $\begin{array}{c}0.82 \\
(0.90-0.82)\end{array}$ & $\begin{array}{c}0.13 \\
(0.20-0.12)\end{array}$ & 0.58 \\
\hline SIC & & & & & & & $\begin{array}{c}0.80 \\
(0.80-0.79)\end{array}$ & $\begin{array}{c}0.78 \\
(0.78-0.74)\end{array}$ & $\begin{array}{c}0.81 \\
(0.81-0.80)\end{array}$ & $\begin{array}{c}0.20 \\
(0.24-0.12)\end{array}$ & $\begin{array}{c}0.21 \\
(0.24-0.12)\end{array}$ & $\begin{array}{c}0.84 \\
(0.84-0.83)\end{array}$ & $\begin{array}{c}0.13 \\
(0.20-0.13)\end{array}$ & 0.54 \\
\hline LIC & & & & & & & & $\begin{array}{c}0.93 \\
(1.05-0.92)\end{array}$ & $\begin{array}{c}0.89 \\
(0.98-0.89)\end{array}$ & $\begin{array}{c}0.15 \\
(0.20-0.15)\end{array}$ & $\begin{array}{c}0.07 \\
(0.13-0.00)\end{array}$ & $\begin{array}{c}0.81 \\
(0.86-0.79)\end{array}$ & $\begin{array}{c}0.20 \\
(0.21-0.20)\end{array}$ & 0.52 \\
\hline LSC & & & & & & & & & $\begin{array}{c}0.86 \\
(0.86-0.85)\end{array}$ & $\begin{array}{c}0.10 \\
(0.13-0.05)\end{array}$ & $\begin{array}{c}0.09 \\
(0.13-0.05)\end{array}$ & $\begin{array}{c}0.80 \\
(0.81-0.79)\end{array}$ & $\begin{array}{c}0.16 \\
(0.17-0.16)\end{array}$ & 0.49 \\
\hline APC & & & & & & & & & & $\begin{array}{c}0.18 \\
(0.25-0.18)\end{array}$ & $\begin{array}{c}0.12 \\
(0.15-0.05)\end{array}$ & $\begin{array}{c}0.83 \\
(0.83-0.82)\end{array}$ & $\begin{array}{c}0.23 \\
(0.25-0.23)\end{array}$ & 0.53 \\
\hline SIM & & & & & & & & & & & $\begin{array}{c}0.71 \\
(0.73-0.70)\end{array}$ & $\begin{array}{c}0.26 \\
(0.34-0.26)\end{array}$ & $\begin{array}{c}0.23 \\
(0.25-0.23)\end{array}$ & 0.30 \\
\hline CEM & & & & & & & & & & & & $\begin{array}{c}0.24 \\
(0.24-0.14)\end{array}$ & $\begin{array}{c}0.18 \\
(0.23-0.18)\end{array}$ & 0.26 \\
\hline LC & & & & & & & & & & & & & $\begin{array}{c}0.23 \\
(0.23-0.23)\end{array}$ & 0.63 \\
\hline CL & & & & & & & & & & & & & & 0.46 \\
\hline
\end{tabular}

Note: AJ: Asiatic Jackal, DH: Dhole, ABB: Asiatic black bear, MSB: Malayan sun bear, LC: Leopard cat, LIC: Large Indian Civet, LSC: Large-spotted civet, APC: Asian palm civet, SIM:

Small Indian mongoose, CL: Clouded leopard, YTM: Yellow-throated marten, HB: Hog badger, SIC: Small Indian Civet, CEM: Crab-eating mongoose 
Table 3. The overlap coefficient $(\Delta)$ calculated using Kernel density functions of large carnivorous mammal species and their prey activity sampled via camera trapping during March, 2017 and February, 2018, in Khao Ang Rue Nai Wildlife Sanctuary, (1=identical activity), with approximate 95\% bootstrap confidence intervals in parentheses.

\begin{tabular}{|c|c|c|c|c|c|c|c|c|c|c|}
\hline Species & GA & BT & SB & $\mathbf{R M}$ & WB & PM & SH & LD & MR & Average \\
\hline $\overrightarrow{\mathrm{AJ}}$ & $\begin{array}{c}0.63 \\
(0.65-0.63)\end{array}$ & $\begin{array}{c}0.63 \\
(0.72-0.63)\end{array}$ & $\begin{array}{c}0.65 \\
(0.67-0.65)\end{array}$ & $\begin{array}{c}0.64 \\
(0.65-0.64)\end{array}$ & $\begin{array}{c}0.58 \\
(0.58-0.57)\end{array}$ & $\begin{array}{c}0.36 \\
036-035)\end{array}$ & $\begin{array}{c}0.72 \\
(081-072)\end{array}$ & $\begin{array}{c}0.62 \\
63-058)\end{array}$ & $\begin{array}{c}0.70 \\
(070-096\end{array}$ & 0.61 \\
\hline DH & $\begin{array}{c}0.42 \\
(0.43-0.42)\end{array}$ & $\begin{array}{c}0.41 \\
(0.43-0.41)\end{array}$ & $\begin{array}{c}0.39 \\
(0.40-0.34)\end{array}$ & $\begin{array}{c}0.80 \\
(0.80-0.78)\end{array}$ & $\begin{array}{c}0.88 \\
(0.92-0.77)\end{array}$ & $\begin{array}{c}0.75 \\
(0.74-0.71)\end{array}$ & $\begin{array}{c}0.40 \\
(0.39-0.34)\end{array}$ & $\begin{array}{c}0.80 \\
(0.84-0.79)\end{array}$ & $\begin{array}{c}0.24 \\
(0.25-016)\end{array}$ & 0.57 \\
\hline $\mathrm{ABB}$ & $\begin{array}{c}0.47 \\
(0.49-0.32)\end{array}$ & $\begin{array}{c}0.47 \\
(0.50-0.39)\end{array}$ & $\begin{array}{c}0.73 \\
(0.81-0.96)\end{array}$ & $\begin{array}{c}0.29 \\
(0.33-0.22)\end{array}$ & $\begin{array}{c}0.22 \\
(0.26-0.18)\end{array}$ & $\begin{array}{c}0.04 \\
(0.08-0.02)\end{array}$ & $\begin{array}{c}0.66 \\
(0.64-0.67)\end{array}$ & $\begin{array}{c}0.28 \\
(0.32-0.26)\end{array}$ & $\begin{array}{c}0.87 \\
(1.06-0.87)\end{array}$ & 0.45 \\
\hline MSB & $\begin{array}{c}0.49 \\
(0.53-0.48)\end{array}$ & $\begin{array}{c}0.46 \\
(0.47-0.45)\end{array}$ & $\begin{array}{c}0.64 \\
(0.65-0.63)\end{array}$ & $\begin{array}{c}0.37 \\
(0.42-0.34)\end{array}$ & $\begin{array}{c}0.36 \\
(0.40-0.35)\end{array}$ & $\begin{array}{c}0.17 \\
(0.20-0.13)\end{array}$ & $\begin{array}{c}0.56 \\
(0.57-0.50)\end{array}$ & $\begin{array}{c}0.48 \\
(0.61-0.48)\end{array}$ & $\begin{array}{c}0.46 \\
(0.47-0.33)\end{array}$ & 0.44 \\
\hline YTM & $\begin{array}{c}0.22 \\
(0.24-0.18)\end{array}$ & $\begin{array}{c}0.22 \\
(0.24-0.21)\end{array}$ & $\begin{array}{c}0.18 \\
(0.20-0.16)\end{array}$ & $\begin{array}{c}0.65 \\
(0.68-0.65)\end{array}$ & $\begin{array}{c}0.75 \\
(0.80-0.74)\end{array}$ & $\begin{array}{c}0.94 \\
(1.05-0.93)\end{array}$ & $\begin{array}{c}0.17 \\
(0.20-0.05)\end{array}$ & $\begin{array}{c}0.57 \\
(0.57-0.43)\end{array}$ & $\begin{array}{c}0.08 \\
(0.12-0.03)\end{array}$ & 0.42 \\
\hline $\mathrm{HB}$ & $\begin{array}{c}0.62 \\
(0.61-0.71)\end{array}$ & $\begin{array}{c}0.56 \\
(0.77-0.55)\end{array}$ & $\begin{array}{c}0.73 \\
(0.91-0.72)\end{array}$ & $\begin{array}{c}0.43 \\
(0.47-0.42)\end{array}$ & $\begin{array}{c}0.38 \\
(0.39-0.37)\end{array}$ & $\begin{array}{c}0.19 \\
(0.20-0.13)\end{array}$ & $\begin{array}{c}0.76 \\
(0.89-0.75)\end{array}$ & $\begin{array}{c}0.48 \\
(0.53-0.44)\end{array}$ & $\begin{array}{c}0.83 \\
(0.88-0.82)\end{array}$ & 0.55 \\
\hline SIC & $\begin{array}{c}0.60 \\
(0.60-0.57)\end{array}$ & $\begin{array}{c}0.64 \\
(0.68-0.63)\end{array}$ & $\begin{array}{c}0.73 \\
(0.73-0.72)\end{array}$ & $\begin{array}{c}0.53 \\
(0.54-0.49)\end{array}$ & $\begin{array}{c}0.46 \\
(0.47-0.41)\end{array}$ & $\begin{array}{c}0.24 \\
(0.25-0.17)\end{array}$ & $\begin{array}{c}0.81 \\
(0.82-0.80)\end{array}$ & $\begin{array}{c}0.56 \\
(0.56-0.55)\end{array}$ & $\begin{array}{c}0.78 \\
(0.78-0.78)\end{array}$ & 0.59 \\
\hline LIC & $\begin{array}{c}0.67 \\
(0.68-0.66)\end{array}$ & $\begin{array}{c}0.68 \\
(0.75-0.67)\end{array}$ & $\begin{array}{c}0.78 \\
(0.88-0.77)\end{array}$ & $\begin{array}{c}0.37 \\
(0.39-0.25)\end{array}$ & $\begin{array}{c}0.29 \\
(0.31-0.18)\end{array}$ & $\begin{array}{c}0.10 \\
(0.13-0.06)\end{array}$ & $\begin{array}{c}0.83 \\
(0.83-0.71)\end{array}$ & $\begin{array}{c}0.43 \\
(0.43-0.26)\end{array}$ & $\begin{array}{c}0.85 \\
(0.91-0.84)\end{array}$ & 0.56 \\
\hline LSC & $\begin{array}{c}0.66 \\
(0.66-0.68)\end{array}$ & $\begin{array}{c}0.66 \\
(0.66-0.65)\end{array}$ & $\begin{array}{c}0.72 \\
(0.72-0.70)\end{array}$ & $\begin{array}{c}0.38 \\
(0.39-0.38)\end{array}$ & $\begin{array}{c}0.31 \\
(0.32-0.31)\end{array}$ & $\begin{array}{c}0.12 \\
(0.13-0.11)\end{array}$ & $\begin{array}{c}0.81 \\
(0.81-0.80)\end{array}$ & $\begin{array}{c}0.40 \\
(0.41-0.31)\end{array}$ & $\begin{array}{c}0.83 \\
(0.84-0.81)\end{array}$ & 0.54 \\
\hline APC & $\begin{array}{c}0.73 \\
(0.75-0.73)\end{array}$ & $\begin{array}{c}0.73 \\
(0.73-0.72)\end{array}$ & $\begin{array}{c}0.80 \\
(0.83-0.79)\end{array}$ & $\begin{array}{c}0.39 \\
(0.40-0.35)\end{array}$ & $\begin{array}{c}0.32 \\
(0.33-0.29)\end{array}$ & $\begin{array}{c}0.14 \\
(0.15-0.12)\end{array}$ & $\begin{array}{c}0.85 \\
(0.89-0.84)\end{array}$ & $\begin{array}{c}0.44 \\
(0.44-0.37)\end{array}$ & $\begin{array}{c}0.80 \\
(0.82-0.80)\end{array}$ & 0.58 \\
\hline SIM & $\begin{array}{c}0.25 \\
(0.29-0.24)\end{array}$ & $\begin{array}{c}0.29 \\
(0.31-0.28)\end{array}$ & $\begin{array}{c}0.25 \\
(0.27-0.24)\end{array}$ & $\begin{array}{c}0.54 \\
(0.55-0.11)\end{array}$ & $\begin{array}{c}0.55 \\
(0.57-0.48)\end{array}$ & $\begin{array}{c}0.69 \\
(0.70-0.58)\end{array}$ & $\begin{array}{c}0.27 \\
(0.28-0.25)\end{array}$ & $\begin{array}{c}0.51 \\
(0.53-0.49)\end{array}$ & $\begin{array}{c}0.08 \\
(0.11-0.03)\end{array}$ & 0.38 \\
\hline CEM & $\begin{array}{c}0.26 \\
(0.27-0.25)\end{array}$ & $\begin{array}{c}0.26 \\
(0.27-0.23)\end{array}$ & $\begin{array}{c}0.20 \\
(0.22-0.15)\end{array}$ & $\begin{array}{c}0.59 \\
(0.59-0.58)\end{array}$ & $\begin{array}{c}0.72 \\
(0.71-0.76)\end{array}$ & $\begin{array}{c}0.89 \\
(0.88-1.06)\end{array}$ & $\begin{array}{c}0.18 \\
(0.22-0.05)\end{array}$ & $\begin{array}{c}0.57 \\
(0.57-0.50)\end{array}$ & $\begin{array}{c}0.08 \\
(0.12-0.03)\end{array}$ & 0.42 \\
\hline LC & $\begin{array}{c}0.72 \\
(0.71-0.73)\end{array}$ & $\begin{array}{c}0.68 \\
(0.68-0.58)\end{array}$ & $\begin{array}{c}0.72 \\
(0.72-0.71)\end{array}$ & $\begin{array}{c}0.55 \\
(0.55-0.52)\end{array}$ & $\begin{array}{c}0.47 \\
(0.47-0.46)\end{array}$ & $\begin{array}{c}0.27 \\
(0.27-0.25)\end{array}$ & $\begin{array}{c}0.80 \\
(0.80-0.79)\end{array}$ & $\begin{array}{c}0.58 \\
(0.58-0.49)\end{array}$ & $\begin{array}{c}0.69 \\
(0.69-0.68)\end{array}$ & 0.61 \\
\hline CL & $\begin{array}{c}0.47 \\
(0.46-0.50)\end{array}$ & $\begin{array}{c}0.45 \\
(0.44-0.47)\end{array}$ & $\begin{array}{c}0.32 \\
(0.31-0.34)\end{array}$ & $\begin{array}{c}0.17 \\
(0.15-0.23)\end{array}$ & $\begin{array}{c}0.16 \\
(0.14-0.22)\end{array}$ & $\begin{array}{c}0.17 \\
(0.15-0.25)\end{array}$ & $\begin{array}{c}0.31 \\
(0.30-0.33)\end{array}$ & $\begin{array}{c}0.30 \\
(0.29-0.32)\end{array}$ & $\begin{array}{c}0.11 \\
(0.09-0.15)\end{array}$ & 0.27 \\
\hline Average & 0.52 & 0.51 & 0.56 & 0.48 & 0.46 & 0.36 & 0.58 & 0.50 & 0.53 & 0.50 \\
\hline
\end{tabular}
Note: GA: Gaur, BT: Banteng, SB: Sambar deer, RM: Red muntjac, B: Wild boar, PM: Pig-tailed macaques, SH: Siamese hare, LD: Lesser mouse deer, MR: Murid rodent , AJ: Asiatic Jackal,
DH: Dhole, ABB: Asiatic black bear, MSB: Malayan sun bear, YYTM: Yellow-throated marten, HB: Hog badger, SIC: Small Indian Civet, LIC: Large Indian Civet, LSC: Large-spotted civet, APC: Asian palm civet, SIM: Small Indian mongoose, CEM: Crab-eating mongoose, LC: Leopard cat, CL: Clouded leopard 
In agreement with past studies, we found carnivores in KARN to be mostly active at night (Simchareon 1990; Van Schaik and Griffiths 1996; Sribuarod 1999; Kanchanasaka 2001; Gonzalez-Maya et al. 2009; Wongchoo et al. 2013; Siripattaranugul et al. 2015a, b), generally observing the carnivores to have the highest activity cycle during 00:0006:00 hrs, and 06:00-08:00 hrs, respectively. The activity of the carnivores was subsequently observed to decrease and reach the lowest values from 12:00-13:00 hrs, with only the dholes, found to have additional activities during the day. Wongchoo et al. (2013) found that the behaviors of the viverrids were nocturnal, with activity observed between 18:00-06:00 hrs, and peaking at dusk from 19:0022:00 hrs, and before dawn, between 04:00-06:00 hrs, respectively. An example $\Delta$ of carnivorous species' daily activities are shown in Figure 3.

In determining the $\Delta$ between carnivorous species in the study area, the $\Delta s$ for nocturnal animals were greater than for the diurnal species (large Indian civet, large-spotted civet, Asian palm civet, and the leopard cat), and had a higher degree of overlap than other carnivorous species. However, the small Indian civet had a lower overlap compared with other carnivores, while the clouded leopard had the lowest overlap due to the very low number of detections. Across carnivorous species, the leopard cat was observed to have the highest $\Delta$ value $(0.63)$, followed by the hog badger (0.58), Asiatic jackal (0.55), small Indian civet (0.54), Asian palm civet (0.53), large Indian civet (0.52), large spotted civet (0.49), Asiatic black bear (0.46), dhole (0.40), Malayan sun bear (0.36), yellow-throated marten (0.31), small Indian mongoose (0.30), crab-eating mongoose (0.26), and clouded leopard (0.17).

In studying the $\Delta$ across all carnivorous and prey species, the carnivore that is the most important hunter in the area was the dhole, with a $\Delta$ value of 0.57 , higher than the average $\Delta(0.46)$ of all 14 species of carnivores. Despite some other carnivores having high $\Delta$ values, they are not direct hunters, therefore their presence in the same area may only be associated with using the same habitat at night; as prey species also forage at night, e.g., gaur, Sambar deer, banteng. This is in contrast to species like the common muntjac and wild boar that are active and present during the daytime. The calculation of the temporal overlap coefficient value of prey species, showed that the prey species, which had the highest $\Delta$ with carnivorous species was the Siamese hare $(0.58)$, followed by the Sambar deer (0.56), murid rodent (0.53), gaur (0.52), banteng (0.51), mouse deer (0.50), common muntjac (0.48), wild boar (0.46) and pig-tailed macaque (0.36), respectively. This demonstrates the importance of prey species in maintaining the society of carnivorous species in the area (see Table 3 ).

A previous study by Lynam et al. (2013), found that the $\Delta$ in clouded leopards and leopard cats were $0.90(0.77$ $0.91)$, however, in this study, the $\Delta$ value for these species was only 0.23 , with the greatest overlap observed in the Malayan sun bear 0.34 (0.34-0.33). Our contrasting findings, are likely related to Lynam et al. (2013) analyzing the combined data from 15 protected areas, while we only considered the area within KARN, identifying leopard cats 115 times and clouded leopards only twice.

In this study, four species of nocturnal viverrids were found, namely the small Indian civet, large Indian civet, large-spotted civet, and Asian palm civet. These species accounted for the highest number of photographs in this study (382 photographs), equating to $48.84 \%$ of the carnivore photographs recorded. These results are similar to Gray and Phan (2011) and Suzuki et al. (2017), who studied camera trap photographs from the Phnom Prich Wildlife Sanctuary and the Chhep Wildlife Sanctuary, Cambodia, respectively. We presently observed the largespotted civet, which is categorized as an endangered species (Timmins et al. 2016), to be the most common out of the 4 species in the KARN area. This species has also been reported in Myanmar, however, they were reported to be very few in number (Zaw et al. 2008), with their presence also recorded in the South-Western part of Cambodia (Holden and Neang 2009) and also in Malaysia (Hamirul et al. 2015). In Thailand, reports show that they are present in both Khao Yai National Park and Thap Lan National Park, but appear uncommon and are near communities (Sukmasuang et al. 2018). Therefore, this study confirms, that alongside Chhep Wildlife Sanctuary in Cambodia, KARN is an important habitat for the largespotted civet, one of the world's endangered species.

The Canid species, including the Asiatic jackal and dhole, was the second most frequent carnivore family found in this study. A total of 235 photographs were captured, accounting for $30.05 \%$ of all the carnivore photographs recorded, with 136 photographs attributed to the jackal, and 99 to the dhole. The dhole is the apex predator in the area (Jenks et al. 2015) as they can hunt large species (Kamler et al. 2015). Reports from Chhep Wildlife Sanctuary suggest that the villagers go into protected areas to harvest forest products and take their pet dogs with them. Dholes have been found dead in traps several times, and there has been an outbreak of canine distemper virus (CDV) in the area, causing dholes to become scarce. However, the presence of dholes in human settlement areas is reportedly less than jackals (Jenks et al. 2015), which is a concern for the management of the protected areas in Thailand, which have found jackals and dholes living near the communities that surround the forest (Jenks et al. 2015).

Four species from the Mustelidae family were found in this study, including the small Indian mongoose, crabeating mongoose, hog badger, and yellow-throated marten. A total of 43 photographs of these species were recorded, which were mainly found during the day, except for the hog badger, which was found at night. The results are in line with previous observations (Grassman et al. 2005; Johnson et al. 2009), which found that hog badgers were mostly nocturnal. In regards to the yellow-throated marten, a camera trap study in the long-term dry evergreen forest in Huai Kha Khaeng Wildlife Sanctuary (Sukmasuang et al. 2018) observed yellow-throated martens to be as active during the day as they were at night. 
In the present study, only two species of bears were found, the Asiatic black bear and the Malaya sun bear, both of which were present during the night. Similar to a report by Suzuki et al. (2017) in Chhep Wildlife Sanctuary, the bears were observed to be few in number compared to other species. In regards to the wild felids, only two species were detected, the clouded leopard and the leopard cat. The clouded leopard was found in only 2 photographs in 1 location; demonstrating the rarity of the clouded leopard in the study area. In comparison, 4 species of wild felids were found in the Chhep Wildlife Sanctuary, Cambodia, including the leopard, clouded leopard, jungle cat and leopard cat (Suzuki et al. 2017). A previous study in the KARN (Sribuarod1999) recorded 23 carnivorous species, including the leopard, tiger and fishing cat, using camera traps and detection of animal signs. However, the previous report (Jenks et al.2012) of the presence of the golden cat in the area, was not confirmed in this study.

In conclusion, this study on the temporal overlap of the wild carnivorous community and their prey in KARN revealed 14 carnivorous species. The majority of the species were nocturnal, except for the dhole, small Indian mongoose, crab-eating mongoose, and yellow-throated marten, whereas the clouded leopard was found to be cathemeral but only 2 pictures were recorded. In studying the $\Delta$ between carnivorous species, it was found that the species with the highest value was the Asian palm civet (0.64) and the lowest was clouded leopard (0.17), with an average $\Delta$ of 0.40 for all of the 14 carnivorous species.

While the carnivorous species and all the prey species had an average $\Delta$ of 0.57 , the species with the highest $\Delta$ in the prey species was the Siamese hare $(0.58)$, and the lowest was pig-tailed macaque (0.36). This shows the importance of the prey species in conserving the carnivore community in the area. Furthermore, the results also show that KARN is an important habitat for the large-spotted civet and dhole, which are on the world's endangered species list. An important threat to the wild species is exposure to diseases from outside of the protected area, via contact with sick domestic animals when coming out to hunt in the community areas. Recommendations for management include strict area protection, especially concerning wildlife poaching in the area, restricting domestic pets into the area, and prohibiting the use of the highway that passes through KARN. In addition, education and publicity about the importance of the wildlife sanctuary as an important habitat for wild carnivores in the eastern forests of Thailand, should be provided. Further studies include the installation of camera traps in this wildlife sanctuary especially in the southern part of the area. Which still cannot be operated due to limitations in accessing the inner area. Studies are also suggested to be undertaken on both the population ecology of important wildlife in the area, with any epidemic of disease in pets and wildlife monitored, in order to prevent disease outbreaks from domestic animals to wildlife.

\section{ACKNOWLEDGEMENTS}

This study was funded by Kasetsart University Research and Development Institute, year 2017, and scholarships for doctoral-level students from the Center for Advanced Studies in Tropical Natural Resources (CASTNaR), Kasetsart University Bangkok, Thailand. The authors would like to thank the former Head of the Forest Biology Department, the Dean of the Faculty of Forestry that supported this study. We thank the Chief of Chachoengsao Wildlife Research Station, Chief of Khao Ang Rue Nai Wildlife Sanctuary and the Head of the Forest Protection Operation Base 1, for providing accommodation and helpful support staff.

\section{REFERENCES}

Azlan MJ. 2009. The use of camera traps in Malaysian rainforests. J Trop Biol Conserv 5: 81-86.

Azlan MJ, Sharma DSK. 2006. The diversity and activity patterns of wild felids in a secondary forest in Peninsular Malaysia. Oryx 40(1): 3641.

Chutipong W, Lynam AJ, Steinmetz R, Savini T, Gale GA. 2014a. Sampling mammalian carnivores in western Thailand: Issues of rarity and detectability. Raffles Bull Zool 62: 521-535.

Chutipong W, Tantipisanuh N, Ngoprasert D, Lynam A, Steinmetz R, Jenks KP. Cutter P. 2014b. Current distribution and conservation status of small carnivores in Thailand: a baseline review. Small Carnivore Conserv 51: 96-136.

Department of National Park, Wildlife and Plant Conservation. 2018. Khao Ang Rue Nai Wildlife Sanctuary. http://web3.dnp.go.th/wildlifenew/animConserveDep.aspx [Thai]

Di Bitetti MS, Di Blanco YE, Pereira JA, Paviolo A, Pe Rez IJ. 2009. Time partitioning favors the coexistence of sympatric crab-eating foxes (Cerdocyon thous) and Pampas foxes (Lycalopex gymnocercus). J Mammal 90: 479-490.

Di Bitetti MS, De Angelo CD, Di Blanco Y E, Paviolo A. 2010. Niche partitioning and species coexistence in a Neotropical felid assemblage. Acta Oecol 36: 403-412.

Fernandez-Duran JJ. 2004. Circular distributions based on nonnegative trigonometric sums. Biometrics 60: 499-503.

Frey S, Fisher JT, Burton AC, Volpe JP. 2017. Investigating animal activity patterns and temporal niche partitioning using camera-trap data: challenges and opportunities. Remote Sens Ecol Conserv 3(3): 123-132.

Gonzalez-Maya JF, Schipper GJ, Benitez A. 2009. Activity patterns and community ecology of small carnivores in the Talamanca region, Costa Rica. Small Carnivore Conserv 41: 9-14.

Gray TNE, Phan C. 2011. Habitat preferences and activity patterns of the larger mammal community in Phnom Prich Wildlife Sanctuary, Cambodia. Raffles Bull Zool 59: 311-318.

Grassman LI Jr, Tewes ME, Silvy NJ. 2005. Ranging, habitat use and activity patterns of binturong Arctictis binturong and yellow-throated marten Martes flavigula in north-central Thailand. Wildl Biol 11: 4957.

Gupta S, Mondal K, Sankar K, Qureshi Q. 2009. Estimation of striped hyena Hyaena hyaena population using camera traps in Sariska Tiger Reserve, Rajasthan, India. J Bombay Nat Hist Soc 106(3): 284.

Hamirul M, Wong CCT, Mohamed A, Lau CF, Mohamad SW, Siwan ES, Rayan DM. 2015. Recent records of large spotted civet Viverra megaspila from Peninsular Malaysia. Small Carnivore Conserv 52 \& 53: 74-83.

Holden J, Neang T. 2009. Small carnivore records from the Cardamom Mountains, southwestern Cambodia. Small Carnivore Conserv 40: 16-21.

Hongliang B, Wang F, McShea WJ, Dajun ZL, Wang SL. 2016. Spatial co-occurrence and activity patterns of mesocarnivores in the temperate forests of Southwest China. PLoS ONE 11(10): 1-15. e0164271.doi:10.1371/journal.pone.0164271 
Jenks K, Chanteap P, Damrongchainarong K, Cutter P, Redford T, Lynam AJ, Howard J, Leimgruber P. 2011. Using relative abundance indices from camera-trapping to test wildlife conservation hypotheses-an example from Khao Yai National Park, Thailand. Trop Conserv Sci 4(2): 113-131.

Jenks KE, Songsasen N, Leimgruber P. 2012. Camera trap records of dholes in Khao Ang Rue Nai Wildlife Sanctuary, Thailand. Canid News 15(4): 1-5.

Jenks KE, Aikens EO, Songsasen N, Calabrese J, Fleming C, Bhumpakphan N, Wanghongsa S, Kanchanasaka B, Songer M, Leimgruber P. 2015. Comparative movement analysis for a sympatric dhole and golden jackal in a human-dominated landscape. Raffles Bull Zool 63: 546-554.

Johnson A, Vongkhamheng C, Saithongdam T. 2009. The diversity, status and conservation of small carnivores in a montane tropical forest in northern Laos. Oryx 43: 626-633.

Kamler JF, Songsasen N, Jenks K, Srivathsa A, Sheng L, Kunkel K. 2015. Cuon alpinus. The IUCN Red List of Threatened Species 2015 e.T5953A72477893. http://dx.doi.org/10.2305/IUCN.UK.20154.RLTS.T5953A72477893.en.

Kanchanasaka B. 2001. Diversity and distribution of carnivores in Khlong Saeng Wildlife Sanctuary, pp. 118-137. In: Wildlife Research Division's Annual Report. National Parks, Wildlife and Plant Conservation Department, Bangkok, Thailand. [Thai]

Karanth U, Sunquist ME. 1995. Prey Selection by Tiger, Leopard and Dhole in Tropical Forests. J Anim Ecol 64 (4): 439-450.

Kawanishi K, Sunquist ME. 2004. Conservation status of tigers in a primary rainforest of Peninsular Malaysia. Biol Conserv 120: 329 344.

Kelly MJ, Holub EL. 2008. Camera trapping of carnivores: Trap Success among Camera types and across Species, and habitat selection by Species, on Salt Pond Mountain, Giles. Northeast Nat 15(2): 249-262.

Laidlaw R, Shaharuddin WNW. 1998. Activity patterns of the Indochinese tiger (Panthera tigriscorbetti) and prey species in Peninsular Malaysia. J Wildl Parks (Malaysia) 16: 85-96.

Lekagul B, McNeely JA. 1988. Mammals of Thailand. Darnsutha Press, Bangkok, Thailand.

Linkie M, Ridout MS. 2011. Assessing tiger-prey interactions in Sumatran rainforests. J Zool 284: 224-229.

Lynam AJ, Jenks K, Tantipisanuh N, Chutipong W, Ngoprasert D, Gale GA, Steinmetz R, Sukmasuang R, Bhumpakphan N, Grassman LI, Cutter P, Kitamura S, Reed DH, Baker MC, McShea WJ, Songsasen N, Leimgruber P. 2013. Terrestrial activity patterns of wild cats from camera-trapping. Raffles Bull Zool 61(1): 407-415

Monterroso P, Alves PC, Ferreras P. 2013. Catch me if you can: diel activity patterns of mammalian prey and predators. Ethology 119 1044-1056.

Network T. 2008. Terrestrial Vertebrate (Camera trap) Monitoring Protocol Implementation Manual. Arlington, VA, USA.

O'Brien TG, Kinnaird MF, Wibisono HT. 2003. Crouching tigers, hidden prey: Sumatran tiger and prey populations in a tropical forest landscape. Anim Conserv 6: 131-139.

Prayoon U, Bhumpakphan N, Sukmasuang R, Kanchanasaka B. 2012 Abundance and habitat suitability of dhole (Cuon alpinus) and their main prey in Thap Lan National Park. J Wildl Thail 19(1): 23-40. [Thai]

Ray J, Sunquist M. 2001. Trophic relations in a community of African rainforest carnivores. Oecologia 127(3): 395-408.

R Development Core Team. 2009. R: A Language and Environment for Statistical Computing. R Foundation for Statistical Computing, Vienna, Austria. http://www.R-project.org.

Ridout MS, Linkie M. 2009. Estimating overlap of daily activity patterns from camera trap data. J Agricu Biol Environ Stat 14: 322-337.

Scognamillo D, Esperanza MI, Sunquist M, Polisar J. 2003. Coexistence of jaguar (Panthera onca) and puma (Puma concolor) in a mosaic landscape in the Venezuelan llanos. J Zool 259(3): 269-279.

Siripattaranukul K, Bhumpakphan N, Sukmasuang R. 2015a. Diversity and abundance of carnivorous mammals in Salakphra Wildlife Sanctuary, Kanchanaburi Province. J Wildl Thail 22(1): 127-140. [Thai]

Siripattaranukul K, Paglia S, Sukmasuang R, Horradee S. 2015b. The study of diversity and abundance of wild animal in Chalerm Rattanakosin National Park by camera trapping. J Wildl Thail 22(1): 91-100. [Thai]

Simchareon S. 1990. Study on Home Range and Daily Activity of Civets: Family Viverridae. [M.Sc. Thesis] Kasetsart University, Bangkok. [Thai]

Sribuarod K. 1999. Diversity, Distribution and Abundance of Mammalian Carnivores in Khao Ang Rue Nai Wildlife Sanctuary. [Thesis] Kasetsart University, Bangkok. [Thai]

Sunarto S, Kelly MJ, Parakkasi K, Hutajulu MB. 2015. Cat coexistence in central Sumatra: ecological characteristics, spatial and temporal overlap, and implications for management. J Zool 296: 104-115. doi: 10.1111/jzo.12218

Sukmasuang R. Kumhom P, McShea WJ, Charaspet K, Pangunta T, Chanachai Y, Bunyavejchewin S, Pongpattananurak N. 2018. Diversity, abundance and activity period of wild mammal in longterm ecological research plot, Huai Kha Khaeng Wildlife Sanctuary using camera trap. In: Wanthongchai K, Sunhornhoa P (eds) Proceedings of the National Technical Forest Conference 22-24 August 2018. Bangkok, Thailand.[Thai]

Suzuki A, Sokha T, Setha T, Akihisa I. 2017. Camera trapping of large mammals in Chhep Wildlife Sanctuary, northern Cambodia. Cambodian J Nat Hist 1: 63-75.

Zaw T, Htun S, Saw HTP, Maung M, Lynam AJ, Latt KT, Duckworth JW. 2008. Status and distribution of small carnivores in Myanmar. Small Carnivore Conserv 38: 2-28.

Timmins R, Duckworth JW, Roberton S, Gray TNE, Willcox DHA, Chutipong W, Long B. 2016. Viverra megaspila. The IUCN Red List of Threatened Species 2016: e.T41707A45220097. http://dx.doi.org/10.2305/IUCN.UK.20161.RLTS.T41707A45220097.en.

Van Schaik CP, Griffiths M. 1996. Activity periods of Indonesian rain forest mammals. Biotropica 28(1): 105-112.

Wongchoo K, Chimchome V, Simcharoen S, Duangchantrasiri S. 2013. Abundance and Distribution of Some Viverrid Species in Huai Kha Khaeng Wildlife Sanctuary. Thai J For 32: 1-9. [Thai]

Zaragozí B, Belda A, Giménez P, Navarro JT, Bonet A. 2015. Advances in integration with GIS. Ecol Inform 30: 6-11. 